\title{
Comparison of Traditional Versus Computer-Based Cognitive Training on Cognition in Elderly with Mild Cognitive Impairment
}

\author{
${ }^{1}$ Dr. Vidhi Shah (PT), ${ }^{2}$ Bhakti Panchal (PT), ${ }^{3}$ Dr. Tushar Palekar (PT), ${ }^{2}$ Padmaja Guruprasad (PT), \\ ${ }^{2}$ Pooja Pokar (PT), ${ }^{4}$ Dr. Kundan Mehta (MD) \\ ${ }^{1}$ Associate Professor, Dr. D. Y. Patil college of Physiotherapy, Pimpri, Pune, Maharashtra, India \\ ${ }^{2}$ PG Student, Dr. D. Y. Patil College of Physiotherapy, Pimpri, Pune, Maharashtra, India \\ ${ }^{3}$ Principal, Dr. D. Y. Patil College of Physiotherapy, Pimpri, Pune, Maharashtra, India \\ ${ }^{4}$ Assistant Professor, Dr. D. Y. Patil Medical college, Pimpri, Pune, Maharashtra, India \\ Corresponding Author : Padmaja Guruprasad (PT)
}

\begin{abstract}
Article Info

Volume 8, Issue 4

Page Number : 180-185

\section{Publication Issue}

July-August-2021

\section{Article History}

Accepted : 02 July 2021

Published : 10 July 2021

Normal ageing cause alterations in the prefrontal cortex, medial temporal lobe system, hippocampus and cerebellum. These changes are the cause of mild cognitive impairment in terms of decreased memory function, reduced speed and executive functions, personality and behavioral disturbances. Computerbased cognitive training is a new tool used for cognitive rehabilitation. This randomized control trial includes 50 subjects, Group A received computerbased cognitive training $(\mathrm{n}=25)$ by using BrainHQ app and Group B received Tradition cognitive training $(\mathrm{n}=25)$ for 3 weeks. Montreal cognitive assessment (MOCA) was taken as outcome measure. The comparison of difference of pre and post MOCA score between Group A and Group B shows $\mathrm{p}=0.002$. Also comparison of MOCA score between male and female of group A shows statistically significant difference with respect to MALE $\mathrm{P}=0.008$ and FEMALE $\mathrm{P}=0.000$.This study provides a strong evidence that Computer Based Cognitive Training showed added improvements in cognition function compared to traditional training.

Keywords : MOCA, prefrontal cortex, medial temporal lobe system, hippocampus, cerebellum
\end{abstract}

\section{INTRODUCTION}

Ageing is the developmental change that occurs in later years of life. Changes due to ageing hampers person's ability to function normally, maintain effortless survival and have high quality of life. In most gerontological literatures, age more than 60 years is considered old age which constitute elderly portion of the population also called senior citizen. According to the $\mathrm{WHO}$, people of $60-74$ years are considered elderly and between 75-84 years are considered old. 
Today people live longer worldwide which causes substantial increase in the ageing population. Dramatic increase in the ageing population also increase health related problems affecting day today activities.

Previous studies have found positive association between cognitive impairment and illness and health status. Depression, insomnia, vascular diseases, hypertension, heart failure, smoking, genetic factor (APOE e4) all show great impact on cognitive performances. Other risk factors for cognitive impairments include advancing age, gender, low level education in early life and living in rural areas. Stronger evidence on cognitive impairments associated with economic status have also been found ${ }^{1}$.

Mild cognitive impairment, also known as minor Neurocognitive disorder is an intermediate stage between clinically diagnosed dementia and complete cognitive intactness ${ }^{2}$. The prevalence of $\mathrm{MCI}$ is greater than dementia. Studies have stated that 8-15\% population with mild cognitive impairment end up with dementia every year and $80 \%$ individuals have dementia within 6 years ${ }^{3}$. Therefore, efforts should be made to keep this people active as much as possible. Early and appropriate intervention in mild cognitive impaired population may reduce further cognitive decline and delay onset of dementia an Alzheimer's disease and thus can reduce the burden on the family members. Therefore, a major research focuses on designing strategies and interventions to slow or reverse age related cognitive decline.

Montreal Cognitive Assessment tool - simple, reliable, and brief test used by health care professionals to assess cognitive performances of a person. It helps in diagnosing people with impairments in their cognitive ability which is mild in nature and Alzheimer's. This is a scale that is a very highly reliable in understanding the deterioration of the cognitive abilities as compared to mini mental scale and other cognitive assessment scales. It helps in understanding and evaluating various components visual perception and high mental functioning tasks, naming the animals, attention, language skills, abstract thinking, any delay in recall. such as and orientation ${ }^{2}$. Total score is 30 . Score $>26-30$ indicates normal, score 19-25 indicates MCI and score <19 indicates Alzheimer's.

It is another common assessment tool used to understand the cognitive functioning of the geriatric population. It was introduced by Folstein et al. in 1975. This test helps is assessing a wide range of cognitive function which includes awareness while performing tasks, orientation, ability to recall, communication skills and visuospatial skills ${ }^{4,5}$ The whole scale is scored between 0-30 where any score more than 24 is normal. Scores ranging from 19-23 are considered with mild cognitive decline. Score ranging from 10-18 are classified as people with moderate cognitive decline and a score $\leq 9$ is considered as part of severe cognitive impairment. ${ }^{4,5}$

\section{Computer based cognitive training}

A recent advance technique used as a training of the cognition is now using computer based program. In various studies that have shown positive effect on cognition in Alzheimer's disease with the use of computer based cognitive training ${ }^{6,7,8}$. Unlike traditional cognitive training, computer-based cognitive training allows to adjust the level according to the patient's cognitive capacity. This is based on the theory of neuroplasticity which goes to explain that the modification of the anatomical and physiological functioning of the brain in response to stimuli which is either environmental and the internal stimuli throughout life.The program which we used in the current study is BrainHQ developed by posit science Inc. San Francisco, CA, USA. A game based computer program developed to train wide range of cognition in of the person. It uses bottom-up 
approach, improving the element building block of perceptual speed and attention, memory and decision making. A program Based on cerebral Neuroplasticity, has shown improvement in cognitive function of community dwelling older adults ${ }^{9,10,11}$.

The focus of the study is to compare the effect of traditional and computer-based cognitive training on cognition in elderly with mild cognitive impairment and to see which training program is more efficient and feasible for elderly. Each training program have their own individual benefits, and thus the current study will help to decide which training program will give most benefit to the patient.

\section{PROCEDURE}

The ethical committee of Dr.D.Y.Patil Vidyapeeth approved the study under the reference No DYPCPT/IEC/30/2020. This randomized control trial study was conducted at Dr D Y Patil hospital and nearby areas like pimple Saudagar, pimple Gurav, kaspate wasti. The Clinical Trials Registry- India (CTRI) number CTRI/2020/12/029497. Total 92 subjects were screened for mild cognitive impairment, eye sight problems, age and literacy. 50 subjects meeting the inclusion exclusion criteria were included in the study and randomly allocated by envelop method. The benefits of the study and the procedure was well explained to the participants in their respective language. Informed consent as taken and signed from all participants. 42 subjects were excluded from the study. 50 subjects then were divided conveniently into two groups:

Group A: Computer based cognitive training group, $\mathrm{n}=25$ Using BrainHQ and

Group B: Traditional cognitive training group, $n=25$ Cognition was assessed by Montreal cognitive assessment scale (MOCA) on first day and scores were recorded in the data recording sheet.

\section{Post training cognition assessment}

- After 9 days of cognitive training, subjects of both the group were assessed for cognition by Montreal cognitive assessment scale on 9th day of training session post training.

- Scores were recorded into the data recording sheet.

There were no dropouts in the study and the data assessor was blinded was blinded about the group allocation in the study.

- Data was analyzed and interpreted.

\section{Data Analysis}

Normality of the data was assessed by using the group A pre and post and Group B pre and post values of MOCA scores using Shapiro wilk test on winpepi software which showed that data was not Normally distributed.

For comparison of MOCA score in group A and B between male and female, Wilcoxon signed rank test was used.

\begin{tabular}{|c|c|c|c|c|c|c|}
\hline $\begin{array}{c}\text { VARIA } \\
\text { BLES }\end{array}$ & & $\begin{array}{c}\text { MEA } \\
\mathrm{N}\end{array}$ & SD & $\begin{array}{c}\text { W } \\
\text { VAL } \\
\text { UE }\end{array}$ & $\begin{array}{c}\text { P } \\
\text { VAL } \\
\text { UE }\end{array}$ & $\begin{array}{c}\text { INTERP } \\
\text { RETATI } \\
\text { ON }\end{array}$ \\
\hline \multirow{6}{*}{$\begin{array}{c}\text { GROUP } \\
\text { A }\end{array}$} & PRE & 22.16 & \pm 1 & \multirow{6}{*}{$\begin{array}{c}4.38 \\
0\end{array}$} & \multirow{6}{*}{$\begin{array}{c}0.00 \\
0\end{array}$} & \multirow{6}{*}{$\begin{array}{c}\text { HIGHLY } \\
\text { SIGNIFI } \\
\text { CANT }\end{array}$} \\
\hline & & & 51 & & & \\
\hline & POST & 27.6 & \pm 1 . & & & \\
\hline & & & $2 J$ & & & \\
\hline & DIFFE & 5.44 & 1.8 & & & \\
\hline & RENCE & & 9 & & & \\
\hline \multirow{4}{*}{$\begin{array}{c}\text { GROUP } \\
\text { B }\end{array}$} & PRE & 20.68 & \pm 1 & \multirow{4}{*}{$\begin{array}{c}4.39 \\
4\end{array}$} & \multirow{4}{*}{$\begin{array}{c}0.00 \\
0\end{array}$} & \multirow{4}{*}{$\begin{array}{c}\text { HIGHLY } \\
\text { SIGNIFI } \\
\text { CANT }\end{array}$} \\
\hline & & & 40 & & & \\
\hline & POST & 24.44 & $\begin{array}{l} \pm 1 \\
22\end{array}$ & & & \\
\hline & $\begin{array}{l}\text { DIFFE } \\
\text { RENCE }\end{array}$ & 3.76 & $\begin{array}{c}1.3 \\
3\end{array}$ & & & \\
\hline
\end{tabular}




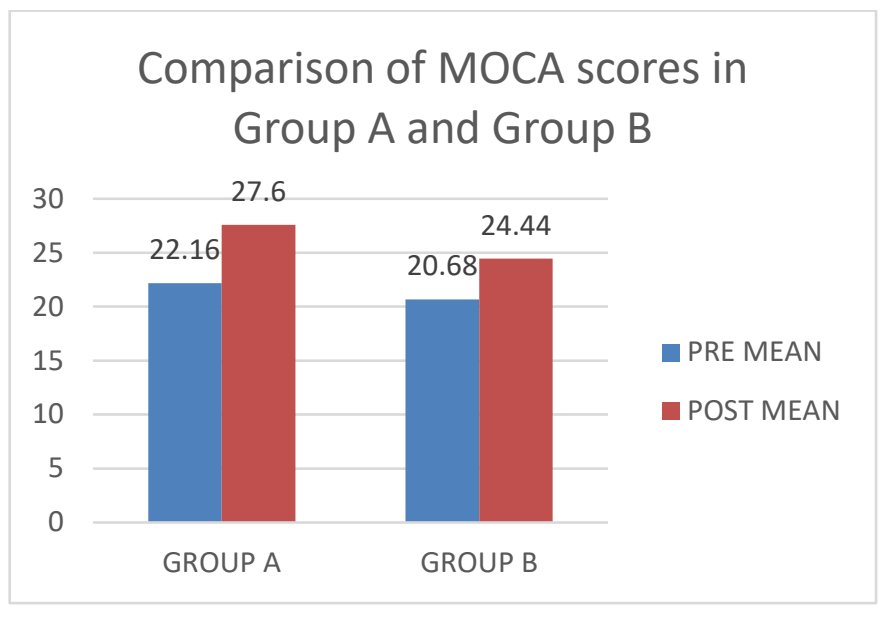

Shows comparison ofMontreal cognitive assessment (MOCA) score between male and female of group A. Within group analysis using Wilcoxon signed rank test showed statistically significant difference with respect to MALE $(\mathrm{P}=\mathbf{0 . 0 0 8})$ and FEMALE $(\mathrm{P}=\mathbf{0 . 0 0 0})$ were higher mean difference reported in Male than Female with mean difference 5.88 (Male) > 5.18 (Female)

\section{DISCUSSION}

This study was conducted as an attempt to find out the effect of Computer Based cognitive training and traditional cognitive training on elder with mild cognitive impairement. Computer Based cognitive training was incorporated by using BrainHq app and traditional cognitive training was given with paper pencil activities like spot the different, solving maze, memory games and mathematical calculations. Study also Aimed to compare both the interventions to check which intervention provided better results in improving cognition in elderly with mild cognitive impairment.

For the same we conducted study in 2 groups with $\mathrm{n}=$ 25 in each group. Group A received computer based cognitive training while Group B received traditional cognitive training for 3 weeks, 3 times in a week. Assessment of cognition was done on first and last day of training using Montreal cognitive assessment scale (MOCA).

Our Study also proved that computer Based cognitive training showed better results. Cognitive function assessed by comparing MOCA scores were more improved in Computer Based Cognitive training when compare to Traditional Cognitive Training group. Computer Based cognitive training have many benefits over traditional cognitive training. Firstly, computerized programs are cost effective for those who have smart phones or computer setups. The participant may not need to travel everyday for the training purpose. Training can be conducted anywhere where the subject resides or is comfortable. Secondly, it can be conducted according to the feasibility of the subject. He or she can decide the time and place for the training. Thirdly, it can be used by those people who cannot participate in more physical intervention strategies like yoga, Pilates, aerobics or strength training. Fourth, it motivates the participant by measuring the progress and immediately providing the feedback for the same. computer based training also gives a time limit to complete the task and also provides scoring and results. This gives participant, the better understanding about his performance and where he lacks and where he needs to work hard for improvement. Computerized training program also provide diverse visual feedback in the form of variety of difference games which keeps the participant more engaged keeping the training playful and exciting which provides a certain level of motivation to stick with the training programs. Last but not the least, computerized training allows customization. Either it automatically sets the level of difficulty or it allows the subject to adjust the difficulty according to the performance. When the participant is doing good, the difficulty level increases and when the participant is not able to cope up with the difficult level, the level can be set low. All this reasons have proven computerized cognitive training to be better than 
traditional cognitive training though both training interventions were effective in enhancing cognitive performances.

Haifeng Zhang et al. studied the effect of multi domain computerized cognitive training on grey matter and its association with neuropsychological performance in patients with Amnestic mild cognitive impairment. After completion of the intervention, they found significant increase in grey matter volume in the right angular gyrus(AG) and other parietal sub areas near the intra parietal sulcus which is responsible for the improvement in the memory and visuospatial memory abilities. And may also delay or slow down the rate of disease progression ${ }^{12}$.

Present study have also found that, on comparison of MOCA scores with gender, males have reported better improvements in the cognitive function after completing cognitive training in both the intervention group I.e computer Based cognitive training and traditional cognitive training. This can be due to the fact that males throughout their life are more engaged in the physical outside world when compare to females who did household chores in general

\section{CONCLUSION}

This study has provided a strong evidence that Computer Based Cognitive Training and Traditional cognitive training both are effective in improving cognition in elderly with mild cognitive decline but Computer Based Cognitive Training showed added improvements in cognition function when compared to tradition cognitive training. Also, on comparison of MOCA scores with Gender, study have found that males have shown more improvements in cognitive performances than females in both the intervention groups.

\section{REFERENCES}

[1]. Kelley BJ, Petersen RC. Alzheimer's disease and mild cognitive impairment. Neurol clin. 2007;25(3):577-609.

[2]. Gorelick PB, counts SE, Nyenhuis D. Vascular cognitive impairment and dementia. Biochim Acta. 2016;1862:860-68.

[3]. Heifeng Zhang, Zhijiang wang. Computerized multi-domain cognitive training reduces brain atrophy in patients with amnestic mild cognitive impairment. Translational psychiatry(2019)9:48

[4]. Harada C N, Natelson Love M C, Triebel K L. Normal cognitive aging. Clin Geriatr Med. 2013;29(4):737-752

[5]. Raz N, Gunning F M, Head D. et al.Selective aging of the human cerebral cortex observed in vivo: differential vulnerability of the prefrontal gray matter. Cereb Cortex. 1997;7(3):268-282.

[6]. Hofman M,Hock C, Muller-Spahn F. Computerbased cognitive training in Alzheimer's disease patients. Ann N Y Acd sci 1996; 777: 249-354.26.

[7]. Schreiber M, Schweizer A, Lutz K, Kalveram KT, Jancke L. Potential of an interactive computerbased training in the rehabilitation of dementia: an initial study. Neuropsychological rehabilitation 1999;9(2):155-67.

[8]. Hofman M, Rosler A, Schwarz W. Interactive computer training as therapeutic tool in Alzheimer's disease. Comer psychiatry 2003; 44(3): 213-9.

[9]. Salat D H, Kaye J A, Janowsky J S. Prefrontal gray and white matter volumes in healthy aging and Alzheimer disease. Arch Neurol. 1999;56(3):338-344

[10]. Pannese E. Morphological changes in nerve cells during normal aging. Brain Struct Funct. 2011;216(2):85-89

[11]. Abdulrahman Al-Thaqib, Fahad Al-sultan. Brain training games enhance cognitive function in healthy subjects. Med sci monic basic res. 2018:24;63-69. 
[12].Elias, L.; Saucier, D. Neuropsychology: Clinical and experimental foundations. Boston: Pearson Education, Inc; 2006.

[13]. Andrew Rosenzweig et al. Montreal cognitive assessment (MOCA) test for dementia. September 23, 2020.

[14]. Ya-yun Lee, Ching-Yi Wu. Evolving methods to combine cognitive and physical training for individuals with mild cognitive impairment: study protocol for a randomised controlled study. 2016; 17: 526

[15].Zelinski EM, spina LM, et al. Improvement in memory with plasticity-based adaptive cognitive training: results of the 3 month followup. J Am geriatric soc. 2011; 59(2): 258-65.

[16]. Smith GE, Housen P, et al. A cognitive training program based on principles of brain plasticity: results from the improvement in memory with plasticity- based adaptive cognitive training (IMPACT) study. J Am geriatr son. 2009; 57(4):594-603.

[17]. Shah V, Tailor R, Palekar TJ, Khandare S. Correlation of cognition with balance, gait measurement and dual task performance in elderly people.

\section{Cite this article as :}

Dr. Vidhi Shah, Bhakti Panchal, Dr. Tushar Palekar, Padmaja Guruprasad, Pooja Pokar, Dr. Kundan Mehta "Comparison of Traditional Versus Computer-Based Cognitive Training on Cognition in Elderly with Mild Cognitive Impairment", International Journal of Scientific Research in Science and Technology (IJSRST), Online ISSN : 2395-602X, Print ISSN : 23956011, Volume 8 Issue 4, pp. 180-185, July-August 2021. Available at doi : https://doi.org/10.32628/IJSRST218424 Journal URL : https://ijsrst.com/IJSRST218424 